\title{
Short Update on Dysbaric Osteonecrosis: Concepts and Decompression Management
}

\author{
Niladri Kumar Mahato ${ }^{1}$
}

\begin{abstract}
Dysbaric osteonecrosis (DON) is caused by inadequate decompression after exposure to very high ambient air pressures. Different pathological events have been shown to occur in conjunction to result in DON. New observations from clinical and experimental data in this field have led investigators to reformat the etiology, pathogenesis and modify existing modalities of treatment of DON. This short review revisits concepts related to pathophysiology of DON occurring as a part of generalized decompression sickness and discusses newer therapeutic insights stemming from recent advancements in DON research.
\end{abstract}

Keywords: Decompression; Dysbarism; Embolism; Hyperbaric Juxta-articular; Metaphysis; Rhizomelic

J Bangladesh Soc Physiol. 2015, December; 10(2): 76-81

For Authors Affiliation, see end of text.

http://www.banglajol.info/index.php/JBSP

\section{Introduction}

D ysbarism or musculoskeletal decompression sickness (DCS) is usually observed in people who undergo deep-sea diving or are exposed to environments of high air pressures ${ }^{1-5}$. Dysbarism or Caisson Disease (CD) is characterized by an array of systemic complications involving soft tissues, the cardio-pulmonary, nervous, renal and musculoskeletal systems when individuals or experimental animals are brought back to normal atmospheric pressures without adequate decompression, even after a single hyperbaric exposure ${ }^{1,6,7}$. Dysbaric osteonecrosis (DON), one of the primary constituents of DCS, has been a subject of prolonged investigations to determine the precise patho-physiology of the affliction, and its prevalence and relationship with DCS. Evidences obtained from patients and data collected from experimental CD animals have

Received June 2015;

Accepted August 2015 pointed towards several etiologies of $\mathrm{DON}^{8-13}$. Given the typical anatomy of vasculature around the end of long bones, it seems plausible that DON is linked to occurrence of micro-embolisms with gas or lipid bubbles following a rapid decrease in surrounding air pressure at those sites $^{3,10,14-17}$. Other investigators have proposed external compression of blood flow in such vessels due to similar bubbles arising in structures around an artery or a vein to induce external pressure. Intra- or extravascular compressions disrupting blood flow usually follow creation of bubbles typically arising from nitrogen gas that is less soluble in the plasma and also expand rapidly to induce morbid symptoms of DCS, once released rapidly from tissues ${ }^{11,12,16,18-20}$.

Recent research suggests that an increased hypercoagulability due to increased levels of serum plasminogen activator inhibitor plays a decisive role in active occurrence and prognosis of DON ${ }^{11,12,19,21}$. The role of lipid dissolution 
in the bone marrow cavity and events of localized and systemic activation of platelet aggregates are also being probed for their purported association with DON. Treatment strategies are being evolved in the lines of our current understanding of DON to prevent its occurrence and to combat long term extremely debilitating osseous damage resulting from rapid and flawed decompression. This paper would briefly review the pathophysiologic concepts associated with DON, discuss current research findings, and outline directions of new therapeutic considera-tions for its management $t^{1,2,5,12,15,19,22,23}$.

\section{Methods}

Primary scientific literature on dysbaric osteonecrosis was accessed as journal articles devoted to clinical studies and experimental models of DON. Online databases were searched using a set of specific search terms and keywords including the ones mentioned in this text. Databases searched included Pubmed $\AA$, Google Scholar ${ }^{\circledR}$ and MEDLINE ${ }^{\circ}$ resources. Special attention was paid to screen and analyze information on the current concepts surrounding the pathogenesis and clinical course of DCS, DON, CD, and current management strategies and future research areas defined in this field.

\section{Discussion}

Features of DON observed in 'dysbarically' injured subjects is thought to be a part of the spectrum of CD symptomatology ${ }^{1,12}$. DON does not feature as an immediate complication in persons presenting with symptoms of generalized decompression sickness ${ }^{9}$. The specific lesions of bone necrosis, per se, are not detected by even sophisticated MRI evaluation at the early stages of the dysbaric injury $8,11,16,22$. However, gas bubbles in the marrow can be picked up using MR techniques ${ }^{8,22}$. Other causes of aseptic bone necrosis being ruled out, lesions specific to DON can take months to develop 1,17,21,23-26. Almost all cases of DCS (including prospective cases of DON) invariably report pain, the 'bends', at their shoulders and /or hips more frequently than other joints ${ }^{6,19,27}$. There are more than a few variables that link DCS to DON and the correlation between these two conditions is controversial ${ }^{1}$. DON can be thought of as a condition that may arise independently with triggering specific patho-physiological events immediately after decompression, or a long term sequel of DCS. In light of this background, it would be worthwhile if one questioned if re-compressive treatment strategies (used for all CD patients) would have the same desired effect for different pathologies operating simultaneously in $\mathrm{CD}^{10,11}$.

This scenario becomes more complicated as newer etiological mechanisms are being identified for DCS -

(i) It all starts with the 'bends': Bends in DCS are characterized by acute pain at or near the limb joints following rapid decompression. Nitrogen is more soluble in fat than in non-fatty tissue. Therefore fat in the bone marrow acts as reservoirs of nitrogen, as more nitrogen gets absorbed within the marrow cavity when a person is subjected to adequate air compression. The bubble threshold is defined as the limit of air compression when fat stores are supersaturated by inert gases beyond which point gas bubbles start to form inside the body, at a given pressure and for a given period of time $\mathrm{e}^{2-4}$. Slow decompression and adequate blood flow in the vessels allows almost all the accumulated gases to diffuse out of the tissues, enter capillary networks and eventually exhaled via the lungs. Since the solubility of nitrogen is poor, rapid decompression creates aggregates of large, symptomatic bubbles. Accordingly, the 'bends' have been thought to occur predominantly due to stretching and irritation of the nerve endings of the marrow sinusoids and the endosteum by the expanding nitrogen bubbles ${ }^{1}$. Bends of DCS is treated by re-compressive hyperbaric oxygen treatment (HBO) that induces a back-diffusion of gases into the tissues thereby preventing embolism. Slowly released gas moving from 
tissues to capillaries do not form bubbles, travel in solution and can be slowly cleared out by the lungs. However, recent investigators have questioned the effectiveness of re-compressive treatment applied across all symptomatic CD patients $5,9,10,28$. They have proposed that application of HBO should be careful and should not result in critically elevated intra-medullary pressure causing death of the marrow cells. Additionally, the intensity of HBO should not be so high that it accentuates pain during treatment and be very specific in the timing of its application and the duration of its exposure to tissues.

(ii) What happens to the fat cells (adipocytes) in rapid decompression ?: Lipid degeneration has been implicated as the starting point of several systemic pathological cascades seen in DCS. Data from animal experiments and human autopsy studies show that rapid decompression results in significant enlargements in the size of adipocytes $^{2,9}$. On further decompression, these cells rupture spilling their contents to forms larger coalesced intracellular aggregates of fat bubbles, in combination with other cell lytic debris. These fat bubbles, in combination with air bubbles, may cause extravascular compression on the tiny epiphyseal or diaphyseal vessels at juxta-articular areas of long bones. Fat bubbles often have been observed to get insinuated into medullary sinusoids locally causing severe intravascular occlusions. These fat emboli cause ischemic necrosis at the end of long bones eventually resulting in bone destruction of $\mathrm{DON}^{2,3,9,12,21}$. As expected, a lot of attention is recently being drawn towards developing imaging strategies that would detect fatty edema of the medulla early in DCS instead of picking up avascular necrosis of DON at a later stage in bone destruction.

Fat droplets released into the bloodstream may result in (a) catastrophic physical embolism and vascular obstruction at vital sites of the body including other bones, (b) activate intrinsic coagulation pathways in blood vessels leading to fatal local or disseminated intravascular coagulation cascades. Even a small increase in intramedullary pressure gradients above the systemic venous pressure may cause fat embolism with its deleterious effects ${ }^{9}$. This may happen with rapid and unregulated HBO therapy, as may be seen with some decompression treatment. Though recompression of a fat coated nitrogen bubble may reduce the size of the bubble by diffusion of the trapped nitrogen (thereby relieving pain symptoms of DCS), the residual fat bubble still remains a potential source of producing DON through occlusion of finer blood vessels. A fair degree of research focus is currently being directed towards understanding different thrombogenetic events related to DON with relation to modulation of lipid and platelet linked enzyme activity ${ }^{9-12}$.

(iii) Role of blood coagulation in DON: There has been a growing body of evidence that connects DON patho-physiology mechanisms to derailment of physiologic intrinsic blood coagulation pathways in the body. Studies related to blood-bubble interface show presence of fibrinogen in the vicinity of the bubble, a decrease in antithrombin III activity, and accelerated platelet aggregation at sites of accumulated intravascular fat and air bubbles ${ }^{9-12}$. Autopsy materials also confirm the presence of thrombi in the osseous blood vessels linked to DON.

(iv) Are free oxygen radicals involved in the pathogenesis ?: Elevated oxidative stress in tissues due to a net rise in free radicals following exposure to wide ranges of altered air pressure has long been suggested to be involved in $\mathrm{DON}^{12,29-32}$. Recent clinical and experimental models of DON have proved that mitochondrial generation of superoxides increase with hyperoxia, hyperbaric environment, hypothermia and exhaustion ${ }^{29,30}$. Though this correlation has not yet been perfectly established, several recent reports indicate that cell death in tissues, including bones, result from free radicals generated from lipid peroxidation, uncoupling of 
mitochondrial oxidative phosphorylation and decomposition of hydrogen peroxide. Experimental models testing the effects of exposure to hyperbaric oxygen or a mixture of gases including Helium as agents of therapeutic HBO applications demonstrate activation of events cited above that leads to tissue damage ${ }^{12,31-32}$. On the basis of this new concept, prophylactic as well as therapeutic administration of free radical scavenging agents is being proposed in the form of vitamins, trace elements and enzymes to counter the short and long term effects of DON.

(v) How does the endocrine system react to elevated pressures ?: It is only recently that researchers have started to find evidence on the etiological role of the endocrine system in dysbaric conditions, specifically in the context of free radical generation in DON. Adrenaline and thyroxin have been found implicated in generation of free radicals even without ambient pressure changes. Oxygen toxicity is reported to be enhanced by thyroxin and adrenalin and both have been documented to increase production of mitochondrial superoxide ${ }^{10,28}$. Enhancement of superoxide levels in plasma following exposure to cold air and secondary to increased air pressure with submersion has been documented in humans. Therapeutic blocking of the effects of circulating catecholamine have been proposed as transient treatment modalities to reduce DON, provided the resultant hypothermia is taken care of otherwise.

(vi) and then there are the Rhizomelic joints: DON probably sets in faster in joints having a predisposition to reduction in venous return from areas of the bone secondary to rise in intramedullary pressure as a result of overall increase in venous stasis. Both the head of the humerus and the femur are two susceptible areas with avascular cartilage covering the spherical heads and the adjoining areas of the metaphysis having (a) no venous channel through these cartilages, (b) narrow metaphyseal necks impeding venous flow and increasing stasis, (c) the heads being spheres, possess lesser surface area in proportion to the volume of cancellous bone inside. A narrow neck in addition, renders the shoulder and the hip joints mechanically inefficient to combat venous stasis when faced with dysbarism ${ }^{21}$. It has been recently proposed that micro-anatomical characterization of joints may explain their predilection for DON. Total sparing of the knee joint from DON may be explained based on this idea.

\section{Conclusion}

Review of recent literatures suggest that though a significant amount of time has passed since the first cases of CD and DON were reported and major advancements in understanding the pathogenesis of DCS achieved, development of effective management and treatment for DCS or DON still eludes us. This seems ironical despite $\mathrm{CD}$ being related to workmen safety issues on one hand and in spite of rapid industrial progress on the other.

\section{Author affiliation}

* 1.Niladri Kumar Mahato,Ohio Musculoskeletal and Neurological Institute, Department of Biological Sciences, Ohio University,Athens, Ohio, 45701. 812603-3307; nm620511@ohio.edu

*For correspondence

\section{References}

1. Gempp E, Blatteau JE, Simon O, Stephant E. Musculoskeletal decompression sickness and risk of dysbaric osteonecrosis in recreational divers. Diving and hyperbaric medicine. 2009;39(4):200-4.

2. Jones JP, Jr., Ramirez S, Doty SB. The pathophysiologic role of fat in dysbaric osteonecrosis. Clin Orthop Relat Res. 1993(296):256-64.

3. Jones JP, Jr. Fat embolism, intravascular coagulation, and osteonecrosis. Clinical orthopaedics and related research. 1993(292):294-308.

4. Jones JP, Jr. Intravascular coagulation and osteonecrosis. Clin Orthop Relat Res. 1992(277): 41-53. 
5. Chryssanthou C. Animal model of human disease: dysbaric osteonecrosis. Am J Pathol. 1981;103(2) :334-6.

6. Phatak UA, David EJ, Kulkarni PM. Decompression syndrome (Caisson disease) in an Indian diver. Ann Indian Acad Neurol. 2010;13(3):202-3.

7. Krebs J, Ferguson SJ, Hoerstrup SP, Goss BG, Haeberli A, Aebli N. Influence of bone marrow fat embolism on coagulation activation in an ovine model of vertebroplasty. J Bone Joint Surg Am. 2008;90(2): 349-56.

8. Dennison S, Fahlman A, Moore M. The use of Diagnostic Imaging for Identifying Abnormal Gas Accumulations in Cetaceans and Pinnipeds. Front Physiol. 2012;3:181.

9. Sharareh B, Schwarzkopf R. Dysbaric osteonecrosis: a literature review of pathophysiology, clinical presentation, and management. Clin J Sport Med. $2015 ; 25(2): 153-61$.

10. Uzun G. Dysbaric osteonecrosis in divers: an editorial perspective. Undersea Hyperb Med. 2014;41(6): 493-4.

11. Uguen M, Pougnet R, Uguen A, Lodde B, Dewitte JD. Dysbaric osteonecrosis among professional divers: a literature review. Undersea Hyperb Med. 2014;41(6):579-87.

12. Kuang XY, Chen LJ, Li HL, Yao F, Xu JM, Huang F, Guo LJ. A study on dysbaric osteonecrosis in caisson workers. Undersea \& hyperbaric medicine : journal of the Undersea and Hyperbaric Medical Society, Inc. 2014;41(3):229-33.

13. Kalsi JS, Abdel-Karim A, Brooke AE. A rare case of osteonecrosis in the premaxilla following meningococcal-induced disseminated intravascular coagulation. J Oral Maxillofac Surg. 2012; 70(12): 2814-8.

14. Zhang Q, Jin SH, Liang WY, Jin Y, Liu XH, Xu Y. [The image diagnosis of dysbaric osteonecrosis]. Zhonghua Lao Dong Wei Sheng Zhi Ye Bing Za Zhi. 2011;29(11):853-5.

15. Kenney IJ, Sonksen C. Dysbaric osteonecrosis in recreational divers: a study using magnetic resonance imaging. Undersea Hyperb Med. 2010;37(5):281-8.
16. Miyanishi K, Kamo Y, Ihara H, Naka T, Hirakawa M, Sugioka Y. Risk factors for dysbaric osteonecrosis. Rheumatology (Oxford). 2006;45(7):855-8.

17. Orces CH. Osteonecrosis: a late sequela of pneumococcal infection and disseminated intravascular coagulation. J Clin Rheumatol. 1999; 5(6):369-71.

18. Carson WK, Mecklenburg B. The role of radiology in dive-related disorders. Mil Med. 2005;170(1): 57-62.

19. Butler WP. Caisson disease during the construction of the Eads and Brooklyn Bridges: A review. Undersea Hyperb Med. 2004;31(4):445-59.

20. Temple JD, Ludwig SC, Ross WK, Marshall WK, Larsen L, Gelb DE. Catastrophic fat embolism following augmentation of pedicle screws with bone cement : a case report. J Bone Joint Surg Am. 2002;84$\mathrm{A}(4): 639-42$.

21. Hutter CD. Dysbaric osteonecrosis: a reassessment and hypothesis. Med Hypotheses. 2000;54(4):585-90.

22. Ozkan H, Uzun G, Yildiz S, Sonmez G, Mutlu H, Aktas S. MRI screening of dysbaric osteonecrosis in hyperbaric-chamber inside attendants. J Int Med Res. 2008;36(2):222-6.

23. Jones GR. Dysbaric osteonecrosis (caisson disease of bone): are active oxygen species and the endocrine system responsible, and can control of the production of free radicals and their reaction products confer protection? Free Radic Res Commun. 1987;4(3): 139-47.

24. Seipolt B, Dinger J, Rupprecht E. Osteonecrosis after meningococcemia and disseminated intravascular coagulation. Pediatr Infect Dis J. 2003;22(11): 1021-2.

25. Salas At, Cabot H. Catastrophic fat embolism following augmentation of pedicle screws with bone cement. J Bone Joint Surg Am. 2003;85-A(8):1613; author reply -4 .

26. Pitto RP, Koessler M, Kuehle JW. Comparison of fixation of the femoral component without cement and fixation with use of a bone-vacuum cementing technique for the prevention of fat embolism during total hip arthroplasty. A prospective, randomized clinical trial. J Bone Joint Surg Am. 1999;81(6): 831-43. 
27. Campbell WN, Joshi M, Sileo D. Osteonecrosis following meningococcemia and disseminated intravascular coagulation in an adult: case report and review. Clin Infect Dis. 1997;24(3):452-5.

28. Cimsit M, Ilgezdi S, Cimsit C, Uzun G. Dysbaric osteonecrosis in experienced dive masters and instructors. Aviat Space Environ Med. 2007; 78(12):1150-4.

29. Bolte H, Koch A, Tetzlaff K, Bettinghausen E, Heller M, Reuter M. Detection of dysbaric osteonecrosis in military divers using magnetic resonance imaging. Eur Radiol. 2005;15(2): 368-75.
30. Laden GD, Grout P. Aseptic bone necrosis in an amateur scuba diver. Br J Sports Med. 2004;3 8(5):E19.

31. Toklu AS, Cimsit M. Dysbaric osteonecrosis in Turkish sponge divers. Undersea Hyperb Med. 2001;28(2): 83-8.

32. Lehner CE, Adams WM, Dubielzig RR, Palta M, Lanphier EH. Dysbaric osteonecrosis in divers and caisson workers. An animal model. Clin Orthop Relat Res. 1997(344):320-32. 